\title{
Context-Aware Human Activity Recognition (CAHAR) in-the-Wild using Smartphone Accelerometer
}

\author{
Yusra Asim, Muhammad Awais Azam, Muhammad Ehatisham-ul-Haq, Usman Naeem, and Asra Khalid
}

\begin{abstract}
Smartphones are a promising platform for continuous monitoring of human behavior. However, the ability to capture people's behavioral patterns in-the-wild is a challenge, as the user's behavior and physical activities can vary, given the variability of settings and environments. Modeling and understanding of human activity in-the-wild must not overlook a user's behavioral context, which is just as crucial as recognizing the range of physical activities. The work in this paper presents a novel framework for context-aware human activity recognition by incorporating human behavioral contexts with physical activities. The proposed framework utilizes a series of machine learning classifiers to validate the efficiency of the proposed method.
\end{abstract}

Index Terms-Activity recognition, accelerometer, behavioral context, context-aware, smartphone, ubiquitous computing

\section{INTRODUCTION}

$\mathrm{U}$ BIQUITOUS computing is a new paradigm, in which the information manipulation is linked with individual actions or objects as encountered anywhere at any time. In recent years, with the rapid advancement in smart gadgets and technologies, the worth of ubiquitous systems has become the key attraction for researchers [1]. These smart devices have been used for enabling a wide range of applications, especially in the area of human activity recognition (HAR). HAR is essential expertise in pervasive computing, which involves detecting the actions or activities of individuals from the sequence of information coming from different sensing modalities. The automatic recognition of human daily living activities has great significance in wide-ranging applications related to robotics, smart surveillance, web-video exploration, and road safety. A lot of research studies have been conducted in the area of HAR using either vision-based [2], [3] or sensorbased approaches [4]-[6]. Recognizing human activities from static images or video sequences is challenging due to certain difficulties, including background mess, restricted occlusion, changes in viewpoint and illumination, and camera motion. Moreover, ubiquitous and in-the-wild monitoring of human activities is not possible with static cameras. These challenges are addressed by the use of miniaturized motion sensors for HAR, including on-body inertial sensors and smartphone sensors. These sensors offer the opportunity of being with the people during the whole day and provide pervasive monitoring of human activities [7]-[9]. Consequently, sensor-based HAR has become significantly crucial for detecting and recognizing in-the-wild human activities.
Most of the existing studies on HAR are developed under controlled settings, where the authors instruct the participants to perform a predefined set of activities to train the system. It causes vile realization of the real-world settings as human physical activity patterns rely heavily on human behavioral context, which varies in different environments. For example, the sitting pattern may be different if a person is in a meeting, or in a car. Similarly, the gait pattern of a person may differ when the person is walking alone or walking with a group of friends. The emotional state of a person also influences his/her physical activity patterns. As a result, the HAR systems trained under controlled settings perform poorly in real-world scenarios. For robust HAR, it is essential to incorporate in-thewild experiments for training and testing of the system. Moreover, it is also necessary to model human behavioral context information along with human activity. Vaizman et al. [10] presented an in-the-wild recognition model for human context, which utilizes the fusion of smartphone sensors (including accelerometer, gyroscope, magnetometer, a location sensor, microphone, and phone state) and watch accelerometer for the recognition of human behavioral context. They used a logistic regression classifier for recognizing multiple single-label contexts for daily living, which include a person's physical activity, phone position, and location. The major limitation of this study is the recognition of single-label human context information, which is not sufficient for better modeling of HAR. It will be more useful if we can recognize multi-label human activity and context information at the same time. For instance, just recognizing a person's physical activity ("such as sitting") or his/her context ("such as indoor") at one time is not enough for fine-grained HAR. However, recognizing a person's physical activity along with the context, such as sitting in a car, is more important and meaningful. Hence, there is a need to be aware of the specific human context along with the physical activities to obtain fine-grained activity information for better decision making. In this aspect, it becomes mandatory to model personal context information in combination with the physical activity simultaneously.

In this study, a novel framework for context-aware human activity recognition (CAHAR) is presented, which combines human behavioral context information with their physical activities for in-the-wild activity and context recognition based on the smartphone accelerometer. The key reason for focusing on the smartphone accelerometer is its better individual performance than the gyroscope and magnetometer sensors, as analyzed in the existing HAR studies [11]. Besides, the fusion of multiple sensors increases the computational complexity of the system and hence becomes infeasible for real-time 


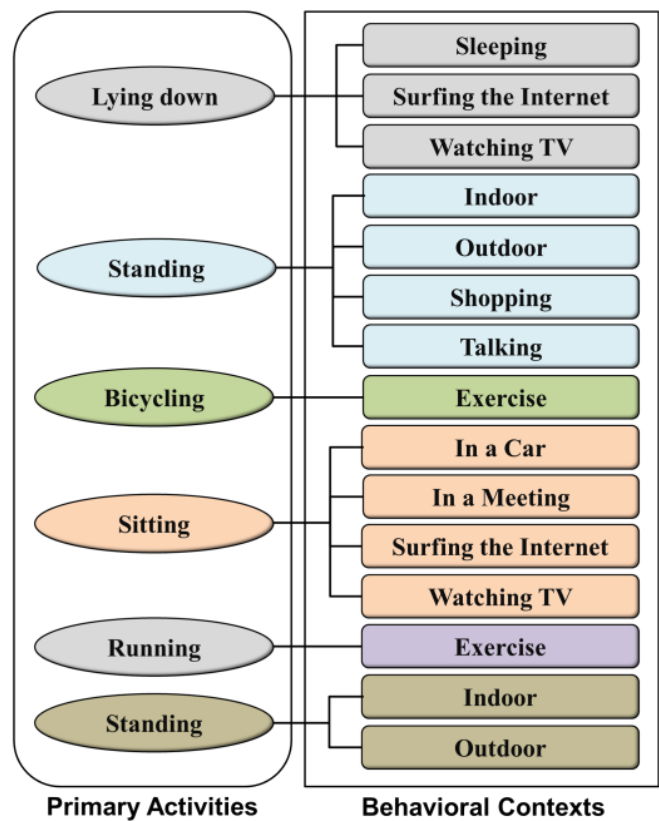

Fig. 1. Primary activities and the corresponding behavioral contexts selected for the proposed CAHAR framework

applications on battery-constrained devices. A public domain dataset, i.e., ExtraSensory [12], is used for validation of the proposed scheme, which contains data (from heterogeneous sensors of smartphone and smartwatch) related to in-the-wild human activities of daily living and their behavioral contexts. Six (06) activities of daily living (ADLs) are considered as primary activities for recognition in the proposed study, which include sitting, standing, lying down, running, bicycling, and walking. For CAHAR, these activities are incorporated with behavioral context information using ten (10) different context labels inline with the ExtraSensory dataset, as shown in Fig. 1. These contexts are defined in terms of location (like indoor and outdoor) and secondary activities (such as talking and shopping). The activity of walking is associated with four contexts, including indoor, outdoor, talking, and shopping, whereas sitting is related to watching $T V$, in a meeting, surfing the internet, and in a car. The activities of bicycling and running are linked with the context of exercise, whereas, for lying down activity, sleeping, surfing the internet, and watching $T V$ contexts are considered. For standing activity, indoor and outdoor contexts are used. By incorporating selected contexts with the primary activities, fifteen (15) different context-aware activities are obtained, which are opted for CAHAR using Random Forest, Bagging, Decision Tree, K-Nearest Neighbors, Support Vector Machine, and Naïve Bayes classifier. For signifying the effectiveness of using a smartphone accelerometer for the proposed scheme, this paper also provides a comparison of the recognition performance of different sensors (phone accelerometer, phone gyroscope, and watch accelerometer) and their fusion for CAHAR.

Major contributions of this research work are as follows:

- A computationally efficient scheme is presented for in-the-wild HAR, which computes simple statistical features from the smartphone accelerometer data only for context-based activity recognition.
- For a better understanding of the human activity, behavioral context parameters (such as location and secondary activity) are incorporated with the primary activity for in-the-wild CAHAR.

- For demonstrating the effect of adding behavioral context information on the recognition of primary activities, two types of experiments are performed, i.e., context-independent HAR and context-aware HAR, and the detailed analysis is presented for each case.

- A detailed comparative analysis is provided regarding the performance of Random Forest, Decision Tree, Bagging, K-Nearest Neighbor, Support Vector Machine, and Naïve Bayes classifier for the proposed CAHAR scheme.

The remaining part of this paper is structured as follows. In Section II, the related work for sensor-based HAR is discussed. In Section III, the proposed CAHAR method is explained in detail. The experimental results and the performance analysis are presented in Section IV. In the end, the research findings are concluded along with the future work in Section V.

\section{RELATED WORK}

Sensor-based HAR has become increasingly significant for human-centric computing. Different types of sensors, mainly ambient sensors, on-body inertial sensors, and smartphone sensors, have been utilized for HAR in different settings. Wearable sensors provide the advantage of being with the people during the whole day and thus facilitate to monitor the daily living activities of human beings continuously. As a result, various research studies emphasized on the utilization of the body motion sensor or wearable inertial sensor for HAR [13]. Bharti et al. [14] proposed an innovative scheme, known as HuMAn, which detects and classifies complex human activities using wearable sensors. The authors extracted timedomain features to train the model using Random Forest and Naïve Bayes classifier and achieved 92\% average accuracy for Random Forest classifier. Anwary et al. [15] presented a gait evaluation system that utilizes Procrustes and Euclidean distance matrix analysis to find out the degree of abnormality in the gait pattern of a person using the wearable accelerometer and gyroscope sensors. The authors collected data from twelve (12) and twenty (20) young and older subjects, respectively, and extracted gait features such as step, swing, stance, and stride for analyzing the gait pattern for any abnormality. HAR based on wearable sensor also helps to detect sudden actions like fall [16] and gives practical guidance about human activities to avoid dangerous behavior. However, wearable sensors often become a source of inconvenience for the users and may lead a person to behave differently, which omits the motive of recognizing natural behavior.

The growing development in the ubiquitous devices (especially smartphones), along with their sensing and networking capabilities, has offered a way to continuously monitor human activities and their behavioral context. A lot of researchers worked on utilizing smartphone inertial sensors for HAR [17]-[19], using Random Forest and Gradient Boosting classifiers. The authors in [20], [21], utilized smartphone 
TABLE I

COMPARISON OF A FEW EXISTING STUDIES FOR SENSOR-BASED HAR

\begin{tabular}{|c|c|c|c|c|c|}
\hline Study / Year & Activity Type & $\begin{array}{l}\text { No. of Activities / } \\
\text { No. of Subjects }\end{array}$ & Classifiers & $\begin{array}{l}\text { Sensing Modality } \\
(\text { Sensor }(\mathbf{s}))\end{array}$ & Accuracy \% \\
\hline [6] / 2016 & Complex ADLs & $13 / 10$ & KNN, DT, NB & $\begin{array}{l}\text { Wearable (A); } \\
\text { Smartphone (A, G) }\end{array}$ & - \\
\hline [10] / 2017 & Behavioral Contexts & $25 / 60$ & Logistic Regression & $\begin{array}{l}\text { Smartphone (A, G, M, GPS, } \\
\text { Wi-Fi); Wearable (A) }\end{array}$ & $87 \%$ \\
\hline [14] / 2019 & Complex at-home ADLs & $21 / 10$ & $\mathrm{NB}, \mathrm{RF}$ & $\begin{array}{l}\text { Wearable (A, G); Ambient } \\
(\mathrm{B}, \mathrm{T}, \mathrm{H}) \text {; GPS; Bluetooth }\end{array}$ & $92 \%$ \\
\hline [18] / 2018 & $\begin{array}{l}\text { Simple ADLs with } \\
\text { Transitional Activities }\end{array}$ & $12 / 30$ & SVM, ANN, DBN & Smartphone (A, G) & $\begin{array}{l}89.61 \% \\
(\mathrm{DBN})\end{array}$ \\
\hline [26] / 2018 & Elderly Activities & $17 / 21$ & SVM & $\begin{array}{l}\text { Wearable (T, A, G, M, B); } \\
\text { Ambient (PIR) }\end{array}$ & $98.32 \%$ \\
\hline [38] / 2017 & Simple ADLs & $06 / 09$ & CNN, RNN & Smartphone & $94.20 \%$ \\
\hline [39] / 2015 & Simple ADLs & $12 / 06$ & $\begin{array}{l}\text { KNN, SVM, RF, GMM, } \\
\text { HMM }\end{array}$ & Wearable & $\begin{array}{l}99.25 \% \\
(\mathrm{KNN})\end{array}$ \\
\hline [40] / 2017 & Firefighter Activities & $17 / 11$ & KNN, GBT, SVM & $\begin{array}{l}\text { Wearable }(\mathrm{A}, \mathrm{G}, \mathrm{M}) \\
\text { Ambient }(\mathrm{B}, \mathrm{H}, \mathrm{T})\end{array}$ & $\begin{array}{l}97.68 \% \\
(\mathrm{GBT})\end{array}$ \\
\hline [41] / 2017 & Simple ADLs & $06 / 20$ & $\begin{array}{l}\text { Deep LSTM Recurrent } \\
\text { Networks }\end{array}$ & Wearable (A, G) & $92 \%$ \\
\hline Proposed & $\begin{array}{l}\text { Context-Aware Activities } \\
\text { (Multi-label Activities) }\end{array}$ & $15 / 60$ & RF, DT, BG & Smartphone (A) & - \\
\hline
\end{tabular}

sensors for user authentication based on activity pattern recognition. Morales and Akopian [22] presented a detailed review of HAR using smartphones and explained the ways of dealing with the unknown phone positions/orientations and selecting the appropriate features, models, classifiers, and metrics for evaluating the usability of a HAR system. A few researchers focused on the combination of smartphone motion sensors and wearable inertial sensors for human activity monitoring [6], [23]-[25]. Y. Wang et al. [26] presented a hybrid sensing approach for elderly activity recognition using the fusion of both wearable and ambient sensors. They evaluated the proposed scheme for seventeen (17) ADLs (from 21 participants) using four different mutual informationbased feature selection methods. Their proposed scheme attained the best recognition accuracy of $98.32 \%$ using the support vector machine classification algorithm with the fusion of all the sensors. Sadiq et al. [27] presented an algorithm for stampede prediction, which uses the fusion of accelerometer, digital compass, and GPS sensors to find the direction and location of people's motion in a crowd situation. Mane and Surve [28] presented a middleware to detect, recognize, and predict human daily life activities using lowlevel contexts, i.e., features, from the sensor data. Khattak et al. [29] proposed HARE, a HAR engine for detecting and recognizing the activities of Alzheimer's disease patients. The authors used video cameras and sensor network to infer the patient's context information (such as high-level physical activity, time, and location) for life care and intelligent decision making.

Recent advances in deep learning techniques have made it possible to extract automatic high-level features from sensor- based data for achieving promising HAR performance. In [30], the authors utilized deep convolutional and LSTM (Long Short-Term Memory) recurrent neural networks (RNN) for multimodal wearable HAR. A few survey articles have also been presented on recent advances in sensor-based HAR using deep learning approaches [31]-[33]. Hammerla et al. [34] presented an exploration of deep, convolutional, and recurrent approaches for HAR based on wearable sensors. They explained how different recurrent approaches could be used for training HAR models in different settings and proposed a regularization scheme that outperforms the existing HAR schemes on the benchmark dataset. The major drawback of deep learning-based approaches is their high computational complexity, which is not feasible for s

Table I provides a comparison of the proposed scheme with some well-known existing schemes for sensor-based HAR. It can be analyzed from the table that the existing sensor-based HAR schemes focus only on the classification of single-label ADLs, without recognizing the user context. However, coinciding recognition of human activities and the associated contexts is mandatory for human behavior analysis and is the core of many context-aware applications, recommender systems, and knowledge-driven systems. Hence, in this research work, we proposed a novel scheme for CAHAR, which recognizes six human activities and the associated contexts in-the-wild, thus enabling recognition of multi-label fine-grained human activities. Moreover, the proposed scheme is computationally advantageous as it is only dependent on the smartphone accelerometer as compared to the existing schemes that mostly rely on the fusion of multiple sensors for achieving effective results. 


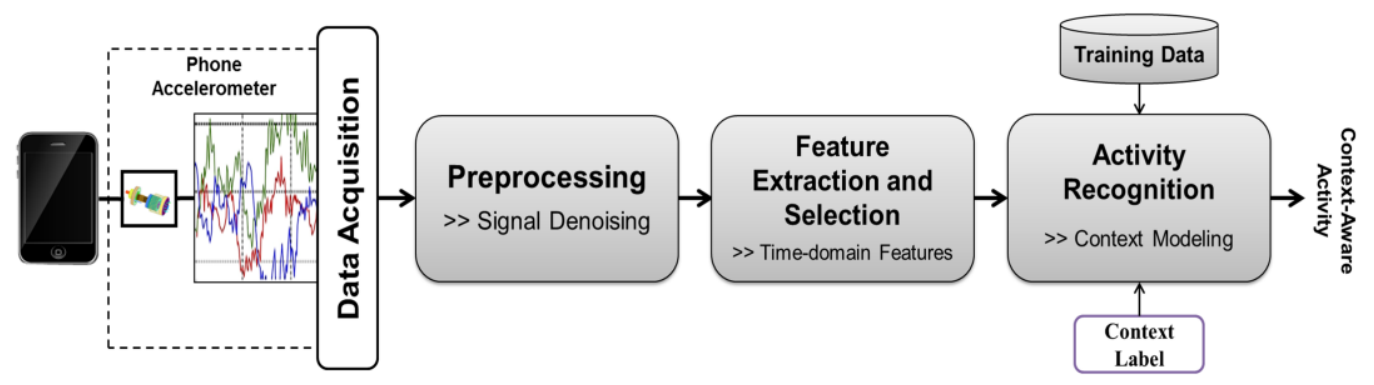

Fig. 2. Methodology for the proposed CAHAR framework

\section{Proposed Methodology}

In this research work, a CAHAR scheme is proposed, which incorporates human behavioral context with human activities for better modeling of human activities in-the-wild. The proposed CAHAR method consists of the following steps: data acquisition and preprocessing, feature extraction and selection, and activity recognition, which are shown in Fig. 2. The necessary details related to each of these steps are covered in the following sections.

\section{A. Data Acquisition, Annotation, and Preprocessing}

The implementation of the proposed method for CAHAR is based on supervised machine learning, which is evaluated on the publicly available ExtraSensory dataset [12] collected in-the-wild from 60 users (26 males and 34 females). Heterogeneous sensors from both smartphone and smartwatch are used for collecting data. These sensors include smartphone motion sensors (such as accelerometer, gyroscope, and magnetometer), audio sensor (microphone), phone GPS, watch accelerometer (with a sampling rate of $25 \mathrm{~Hz}$ ), and watch compass. In our proposed CAHAR scheme, we only used raw data from the accelerometer sensor of the smartphone, which was collected at a sampling rate of $40 \mathrm{~Hz}$.

The ExtraSensory dataset comprises of six primary activities (e.g., lying down, sitting, walking, standing, running, and bicycling) per user. In addition, the dataset also entails a large number of binary context labels (consisting of secondary activity label and the user's context information) per user, which represent the overall behavioral context of the user. However, the contexts labels are not consistent for all the users. For the implementation of the proposed scheme, we incorporated overall ten (10) different context labels with six (06) primary activities (as shown in Fig. 1) in different combinations. The association between the selected primary activities and the corresponding behavioral contexts is made owing to their higher frequency of occurrences together in the ExtraSensory dataset. In this aspect, for each user, we counted the number of different context labels that occur in pairs with each primacy activity. In the end, we pick fifteen (15) most frequent activity-context pairs as context-aware activities, which have at least 500 instances across multiple users. Hence, this entire process of data annotation is systematic and reproducible.

For mitigating the effect of any unwanted noise from the acquired raw data, we applied a time-domain average smoothing filter (with order $[1 \times 3]$ ) on the raw accelerometer data. As the noise encompasses high-frequency elements, hence, a smoothing filter can minimize the effect of abrupt spikes in the signal, which are generated owing to the system or participant noise. The selection of window size is an essential factor for data segmentation and affects the final recognition results. The existing studies [6], [11] have revealed that a 5-second time window is enough to recognize simple and repetitive human activities, such as walking or running. However, complex HAR underperforms when a small window length is used. Hence, for complex activities, a window size from $15 s$ to $30 s$ has been used by the researchers [6], which has shown excellent recognition performance. The proposed CAHAR scheme aims to recognize multi-label context-aware activities, which are complex and chaotic in nature. Furthermore, the ExtraSensory dataset used for the validation of our proposed scheme encompasses presegmented data chunks of 20 -seconds in length. Hence, in line with the pilot study [12], we used the same window length for the proposed scheme to obtain successful recognition results.

\section{B. Feature Extraction and Selection}

Feature extraction is a crucial step in any classification task as the recognition performance is directly affected by the type of attributes extracted from the raw data. As the proposed CAHAR scheme aims to effectively discriminate between the varying physical activity patterns in different contexts, hence it is necessary to choose a robust set of features for activity classification. For this purpose, we extracted eighteen (18) time-domain features from the preprocessed accelerometer data, which have revealed efficient performance in HAR related studies [10], [11], [35]. These features provide essential signal traits that are significant in recognizing human activities based on sensor data. For example, maximum and minimum latency represents the time when the signal reaches its maximum/minimum value, kurtosis is a measure of the tailedness of the signal probability distribution, signal percentile depicts information about the rank of the signal, first or second difference highlights the sharp changes in the signal values, and entropy measures the rate of change in the signal. Table II provides the mathematical description of the features extracted in this study for CAHAR. These features were extracted along each axis of the 3D sensor using a 20second fixed-length window with no overlapping samples. After extracting these features from the preprocessed raw data, we applied a supervised correlation-based feature subset selection (CfsSubetSel) [36] method to eliminate the redundant features. The final subset of the selected features is then used for activity recognition tasks. 
TABLE II

LIST OF TIME DOMAIN FEATURES EXTRACTED FOR CAHAR

\begin{tabular}{|c|c|}
\hline Feature & Mathematical Equation \\
\hline Maximum Amplitude & $X_{\max }=\max (\mathrm{s}(\mathrm{n}))$ \\
\hline Minimum Amplitude & $X_{\min }=\min (\mathrm{s}(\mathrm{n}))$ \\
\hline Maximum latency & $n_{X_{\max }}=\left\{n \mid s(n)=X_{\max }\right\}$ \\
\hline Minimum latency & $n_{X_{\min }}=\left\{n \mid s(n)=X_{\min }\right\}$ \\
\hline Arithmetic Mean & $\bar{\mu}=\frac{1}{N} \sum_{n=1}^{N} s(n)$ \\
\hline Kurtosis & $K=\frac{E\left[(s(n)-\bar{\mu})^{4}\right]}{E\left[(s(n)-\bar{\mu})^{2}\right]^{2}}$ \\
\hline Variance & $\sigma^{2}=\frac{1}{N} \sum_{n=1}^{N}(\mathrm{~s}(n)-\bar{\mu})^{2}$ \\
\hline Standard deviation & $\sigma=\sqrt{\frac{1}{N}} \sum_{n=1}^{N}(s(n)-\bar{\mu})^{2}$ \\
\hline Third Moment & $m_{3}=\mathrm{E}\left[(\mathrm{s}(\mathrm{n})-\bar{\mu})^{3}\right]$ \\
\hline Fourth Moment & $m_{4}=\mathrm{E}\left[(\mathrm{s}(\mathrm{n})-\bar{\mu})^{4}\right]$ \\
\hline Skewness & $S=\frac{m_{3}}{m_{3}^{3 / 2}}$ \\
\hline $\begin{array}{l}\text { Mean of absolute values } \\
\text { of first difference }\end{array}$ & $u_{\nabla}=\frac{1}{N} \sum_{n=1}^{N}|s(n)-s(n-1)|$ \\
\hline $\begin{array}{l}\text { Mean of absolute values } \\
\text { of second difference }\end{array}$ & 1) $-2 s(n)+s(n-1)$ \\
\hline $25^{\text {th }}$ - percentile & $r=\operatorname{round}(0.25 * N)$ \\
\hline $50^{\text {th }}$-percentile & $r=\operatorname{round}(0.50 * N)$ \\
\hline $75^{\text {th }}$-percentile & $n_{75}=s(r), \quad r=\operatorname{round}(0.75 * N)$ \\
\hline Energy & $e=\frac{1}{N} \sum_{n=1}^{N}\left(s(n)^{2}\right.$ \\
\hline Value entropy & $H(s(n))=-\sum_{i=1}^{N} P_{i}\left(s(n) \log _{2} P_{i}(s(n))\right.$ \\
\hline
\end{tabular}

* $s(n)$ represents the acceleration signal along $\mathrm{x}, \mathrm{y}$, or $\mathrm{z}$-axis of the accelerometer, $N$ is the length of sequence $s(n), E$ symbolizes the expected value, $r$ is the rank of the signal $s(n)$, and $P_{i}$ is probability value.

\section{Activity Recognition}

The last step of the proposed method is the automatic recognition of context-aware human activities based on the final set of features obtained from the smartphone accelerometer data. Identifying context-aware activities inthe-wild is challenging due to the prodigious inconsistencies in the human activity patterns, occurring as a result of diverse behavioral contexts. However, these contexts provide more ample and fine-grained information about human activities, which is necessary for efficient activity modeling and a better understanding of human behavior. For this purpose, the proposed scheme explicitly models six (06) primary activities with overall ten (10) behavioral contexts to learn and identify fifteen (15) context-aware activities (as shown earlier in Fig. 1) based on the smartphone accelerometer data. A set of machine learning classifier is used for the purpose of activity recognition. These classifiers are trained separately for different behavioral contexts associated with each primary activity to learn and recognize the selected primary activities along with their behavioral contexts. In this way, the proposed scheme provides the notion of CAHAR and offers finegrained information about in-the-wild human activities.
Furthermore, for analyzing the effect of behavioral context modeling on HAR performance, two types of experiments are conducted for activity recognition, i.e., context-independent HAR (primary activity recognition) and context-dependent HAR (context-aware activity recognition). For contextindependent HAR, a conventional HAR approach is followed, where the selected classifiers are trained to recognize only six (06) context-independent activities that are chosen as primary activities in the proposed scheme. These activities are recognized in-the-wild as single-label activities without incorporating any context information. However, in the case of context-dependent HAR, the proposed idea of CAHAR is implemented, which involves adding behavioral context information with the primary activities for context-aware activity recognition. The detailed analysis regarding both types of experiments is provided in the results section.

\section{EXPERIMENTAL RESUltS AND PERFORMANCE ANALYSIS}

This section describes the experimental setup and provides the numerical results obtained for the proposed CAHAR scheme. Detailed discussion and analysis are provided regarding CAHAR results.

\section{A. Experimental Setup}

\section{1) Machine Learning Classifiers and Hyperparameters}

For validating the proposed methodology for activity recognition, six (06) different machine learning classifiers are employed, which included Random Forest (RF), Decision Tree (DT), Bagging (BG), K-Nearest Neighbors (KNN), Support Vector Machine (SVM), and Naïve Bayes (NB). The hyperparameters relating to these classifiers are tuned such that the training error of each classifier is minimized. A random tree with a base learner is utilized for RF classifier, where the number of iterations is set to 100. A fast decision tree learner (with the number of folds equal to 3 ) is employed for BG classifier, which builds a tree from information variance and prunes it using back fitting. A J48 pruned tree is used for DT classifier with three folds. In the case of KNN classifier, Euclidean distance is used for similarity measure, and the nearest neighbor parameter is set equal to 1 (i.e., $\mathrm{K}=1$ ). For SVM classifier, the one-vs.-one approach is used with sequential minimal optimization (SMO) [37] algorithm.

\section{2) Performance Metrics and Validation Scheme}

The recognition performance of the selected classifiers is measured in terms of accuracy, precision, sensitivity (recall), specificity, and f-measure (harmonic mean of precision and recall). However, these metrics, particularly precision and $f$ measure, are highly sensitive to rare-labels (in the case of the imbalanced dataset), which may lead to biased recognition results for some activities. As the ExtraSensory dataset is collected in-the-wild; hence, the sample distribution for different activities is not uniform. Hence, to avoid any unfairness in the recognition results, balanced accuracy (BA) is taken as a key performance indicator in this study, which does not suffer from the rare-labels issue and provides fair results in the case of imbalanced class distribution [10]. The BA value is calculated by averaging the value of sensitivity and specificity. 
TABLE III

PERFORMANCE COMPARISON OF RF, BG, DT, KNN, SVM, AND NB CLASSIFIER FOR THE PROPOSED CAHAR METHOD

\begin{tabular}{llcccc}
\hline \hline Experiment Type & Classifier & Accuracy & Precision & Sensitivity & Specificity \\
\hline & Random Forest (RF) & 0.871 & 0.840 & 0.754 & 0.846 \\
& Bagging (BG) & 0.830 & 0.791 & 0.698 & 0.845 \\
Context-independent HAR & Decision Tree (DT) & 0.790 & 0.675 & 0.675 & 0.843 \\
(06 Primary activities) & K-Nearest Neighbors (KNN) & 0.595 & 0.454 & 0.431 & 0.898 \\
& Support Vector Machine (SVM) & 0.584 & 0.552 & 0.275 & 0.912 \\
& Naïve Bayes (NB) & 0.570 & 0.309 & 0.311 & 0.862 \\
\hline & Random Forest (RF) & 0.840 & 0.795 & 0.617 & 0.941 \\
& Bagging (BG) & 0.798 & 0.795 & 0.537 & 0.939 \\
Context-dependent HAR & Decision Tree (DT) & 0.592 & 0.760 & 0.525 & 0.939 \\
(15 Context-aware activities) & K-Nearest Neighbors (KNN) & 0.528 & 0.198 & 0.153 & 0.955 \\
& Support vector Machine (SVM) & 0.542 & 0.223 & 0.117 & 0.960 \\
& Naïve Bayes (NB) & 0.502 & 0.288 & 0.277 & 0.952 \\
\hline \hline
\end{tabular}

For training and testing of the proposed CAHAR scheme using the selected classifiers, a 10-fold cross-validation scheme is used, where $90 \%$ data is used for classifier training, and the remaining $10 \%$ data is used for testing the classifier. Ten different iterations are performed for training and testing the proposed system based on ten random splits of data obtained with 10 -fold cross-validation. The average results of all the iterations are provided in the next section.

\section{B. Experimental Results and Discussion}

As discussed before in Section III.C, HAR experiments are conducted for the proposed scheme in two different phases, i.e., context-independent HAR and context-dependent HAR. For this purpose, the final subset of features obtained after applying CfsSubetSel is passed as input to the selected classifiers for HAR. The feature selection process is applied only for context-aware activity recognition, where a subset of eight (08) distinct features is obtained for smartphone accelerometer data. These features include minimum amplitude, mean, maximum latency, third moment, kurtosis,

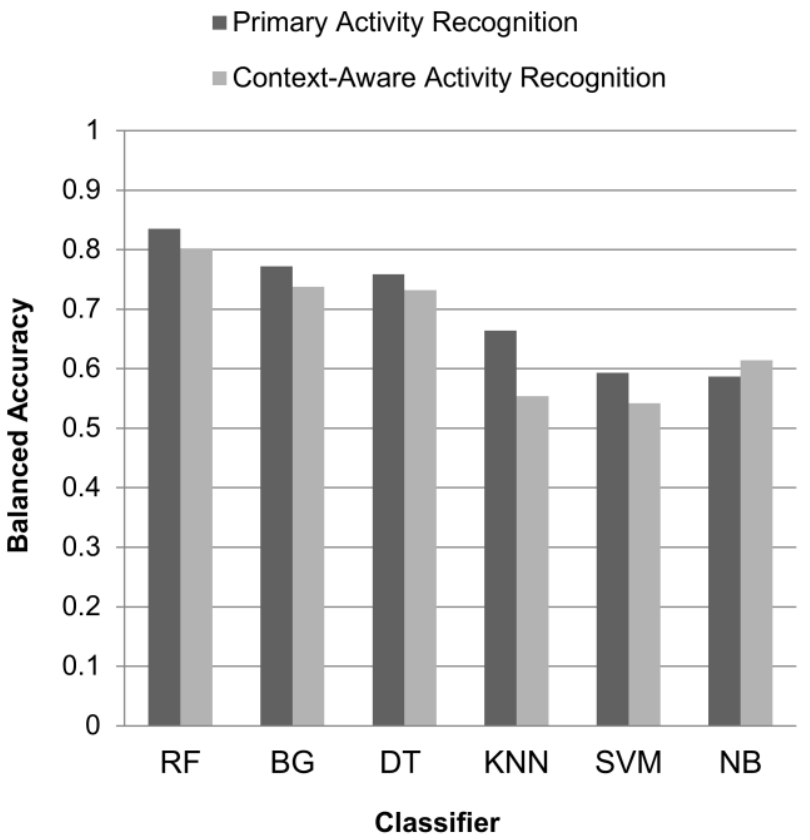

Fig. 3. Comparison of balanced accuracies achieved for primary activity recognition and context-aware activity recognition using the selected classifiers. $50^{\text {th }}$-percentile, mean of absolute values of the first difference, and value entropy. The same set of final features is used for context-independent HAR as well. Thus, the size of the final feature obtained for each data chunk is $[1 \times(8 \times 3)]=[1 \times 24]$ for both types of HAR experiments.

Table III compares the performance of the selected classifiers for context-independent and context-dependent HAR in terms of accuracy, precision, sensitivity, and specificity. It can be easily observed from the table that RF classifier provides better results for both types of experiments based on these performance measures. Fig. 3 compares the balanced accuracies achieved for primary activity recognition (i.e., context-independent HAR) and context-aware activity recognition (i.e., context-dependent HAR) using different classifiers. It can be analyzed from the figure that RF classifier achieves the best average BA of $80 \%$ in recognizing six primary activities for context-independent HAR experiments. For the same experimental setting, BG and DT classifiers achieve an average BA of $77.2 \%$ and $75.9 \%$, respectively, which is $2.8 \%$ and $4.1 \%$ lesser than that obtained for RF classifier. Likewise, KNN, SVM, and NB classifiers achieve an average BA of $66.4 \%, 59.3 \%$, and $58.7 \%$, respectively, which is $13.6 \%, 21.7 \%$, and $22.3 \%$ smaller than the best BA value obtained using RF classifier. Moreover, the average BA values achieved for context-dependent HAR using RF, BG, DT, KNN, SVM, and NB classifier are $77.9 \%, 73.8 \%, 73.2 \%$, $55.4 \%, 54.2 \%$, and $61.4 \%$ respectively, which states that RF classifier performs better than the rest of classifiers. Moreover, SVM, KNN, and NB classifiers provide the lowest recognition accuracies for the proposed scheme, which shows their inability to handle in-the-wild data effectively. Generally, SVM provides excellent recognition performance when the margin of separation between the target classes is higher, and the number of dimensions is more than the number of samples. As in-the-wild human activity patterns entail significant variations, noisy data, and outliers; hence, SVM classifier does not perform well as the target classes become overlapping. $\mathrm{KNN}$ is a lazy-learner and cannot generalize the training model before seeing the test data, hence not useful for classifying the real-time data. Similarly, NB is a simple probabilistic classifier that works on the principle of Bayes theorem and cannot handle variations in human activity patterns in diverse behavioral contexts, thus produce insufficient recognition results. 
As compared to DT classifier, RF performs well as it uses a combination of decision trees to improve the classification performance. RF randomly selects the partition nodes for constructing multiple decision trees, and then uses a majority voting criterion for assigning new examples to a specific class. Moreover, RF classifier improves the variance between different classes by reducing the correlation between multiple trees. On the other hand, BG improves variance by averaging the outcome from multiple fully grown trees on different variants of the training set. RF only uses a subset of randomly selected features out of the total features and uses the best split feature to split each node in a tree. However, BG utilizes all the features for splitting a tree node, which affects its recognition performance as well. As a result, RF generally performs better than DT and BG classifier in most cases, as depicted by the existing HAR studies. The experimental results endorse the efficacy of using RF classifier for the proposed CAHAR method.

By comparing the average recognition results achieved for context-independent and context-dependent HAR in Fig. 3, it can be observed that context-independent activities are recognized with a better BA as compared to context-aware activities. It is due to the fact that it is more natural to discriminate between different primary activities (such as walking, bicycling, and sitting) as the activity patterns are entirely different as a result of significant inter-class variation even in diverse contexts. However, differentiating between the changing patterns of the same activity in different contexts is difficult as the margin of error is minimal due to small intraclass variation. This variation occurs due to the reason that human activity patterns are dependent on their behavioral context, and change on account of varying context in-the-wild. Moreover, different persons have their own way of interacting and behaving in different contexts, which makes it tough to model context-aware human activities in-the-wild. As a result, the addition of user behavioral context with human activities makes the recognition task more laborious and difficult. Nevertheless, the proposed scheme provides effective recognition results for CAHAR experiments as well, where fifteen activities are recognized on account of only $2.1 \%$ decrease in the overall average BA value as compared to that achieved for context-independent recognition of six primacy activities.

Fig. 4 and Fig. 5 provide the balanced accuracies achieved pertaining to each individual activity for context-independent and context-dependent HAR, respectively, using RF classifier. Table IV and Table $\mathrm{V}$ present in depth the numerical values for other performance metrics related to each individual activity for context-independent and context-dependent HAR, respectively. It can be observed from Fig. 4 that it is easier to recognize dynamic activities, such as running $(\mathrm{BA}=84.2 \%)$ and bicycling $(\mathrm{BA}=84.8 \%)$, in-the-wild as compared to static activities, such as standing (BA=79.3\%), and lying down $(\mathrm{BA}=70.3 \%)$ activities. Static activities usually do not exhibit any significant motion due to which it becomes difficult to distinguish between these activities in-the-wild on the basis of phone accelerometer without having any prior knowledge of the user's context. Hence, in-the-wild recognition of the context-independent static activities becomes difficult as compared to the dynamic activities.

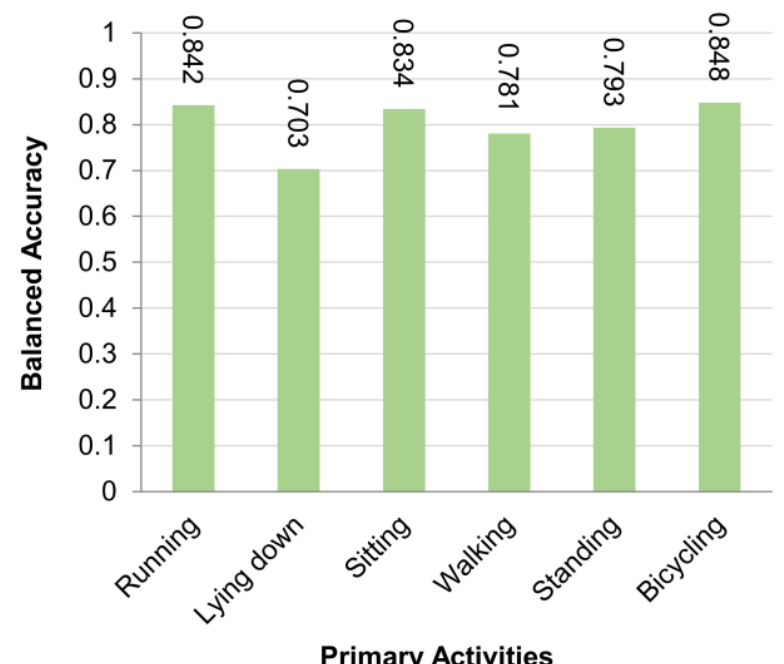

Fig. 4. Comparison of individual BA values achieved for six primary activities using RF classifier.

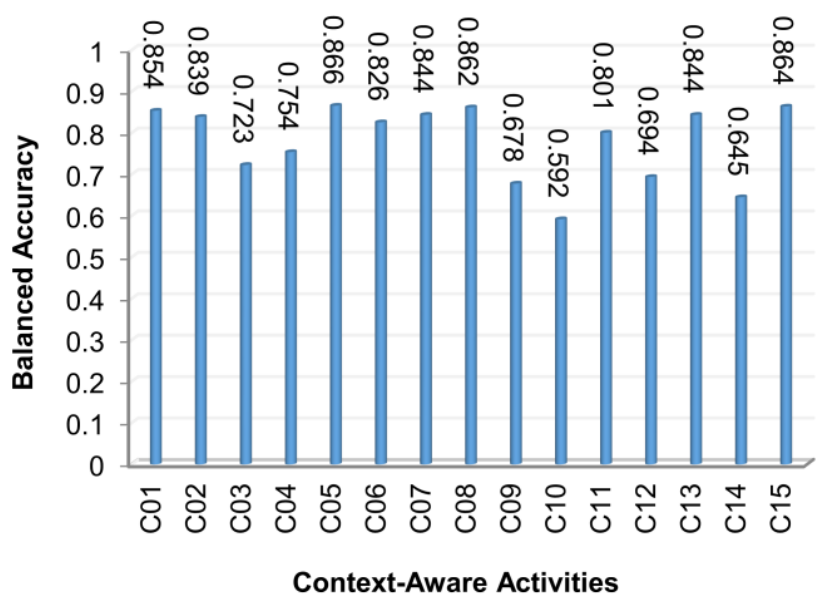

Fig. 5. Comparison of individual BA values achieved for fifteen contextaware activities using RF classifier. Table IV provides the labels for the activitv codes related to these context-aware activities.

TABLE IV

INDIVIDUAL RECOGNITION PERFORMANCE ACHIEVED FOR EACH PRIMARY ACTIVITY USING RANDOM FOREST CLASSIFIER

\begin{tabular}{lccccccc}
\hline \hline Primary Activity & Code & \# of Instances & Accuracy & Precision & Sensitivity & F-measure & Specificity \\
\hline Running & A01 & 1052 & 0.998 & 0.963 & 0.688 & 0.802 & 0.995 \\
Lying down & A02 & 83811 & 0.952 & 0.956 & 0.955 & 0.956 & 0.450 \\
Sitting & A03 & 33490 & 0.916 & 0.772 & 0.877 & 0.821 & 0.791 \\
Walking & A04 & 8817 & 0.961 & 0.685 & 0.597 & 0.638 & 0.964 \\
Standing & A05 & 20946 & 0.928 & 0.763 & 0.688 & 0.724 & 0.899 \\
Bicycling & A06 & 4953 & 0.989 & 0.906 & 0.720 & 0.802 & 0.976 \\
\hline \hline
\end{tabular}


In the case of context-aware activities, it can be seen from Fig. 5 that the activity of sitting in a meeting is best recognized with a BA of $86.6 \%$. Similarly, the values of BA obtained for the activity of sitting with other contexts such as in a car, surfing the internet, and watching $T V$ are also higher than $80 \%$, which shows that recognition of context-dependent activities relating to the sitting activity is more relaxed when compared to other static or dynamic activities. The recognition of lying down activity when surfing the internet is more comfortable as compared to other contexts, such as watching $T V$ and sleeping. Likewise, standing indoor is easier to recognize than standing outdoor. Recognition of the dynamic activity of walking is poorly affected by different behavioral contexts. It can be analyzed from Fig. 5 and Table V that context-aware activities of walking indoor, walking while shopping, and walking while talking are challenging to recognize owing to the vast diversity in the activity patterns among different users. Moreover, any change in the user's context (such as secondary activity or phone position) adversely affects the walking activity pattern, and there is a great likelihood for the walking activity to get misclassified with some other dynamic activity in-the-wild, such as running or bicycling. The average recognition accuracy of lying down and sitting activities is increased to $77.2 \%$ and $85 \%$, respectively, in the case of context-dependent recognition. Conversely, for walking and standing activities, the average BA values achieved for context-dependent HAR are reduced to $69.1 \%$ and $74.45 \%$, respectively. Generally, the activities of bicycling while exercise, sitting in a meeting, and sitting while surfing the internet are more comfortable to recognize using a smartphone accelerometer as depicted by the results presented in Table V.

TABLE V

INDIVIDUAL RECOGNITION PERFORMANCE ACHIEVED FOR EACH CONTEXT-AWARE ACTIVITY USING RANDOM FOREST CLASSIFIER

\begin{tabular}{|c|c|c|c|c|c|c|c|}
\hline Context-aware Activity & Code & \# of instances & Accuracy & Precision & Sensitivity & F-measure & Specificity \\
\hline Running while exercise & $\mathrm{C} 01$ & 1052 & 0.997 & 0.953 & 0.713 & 0.815 & 0.995 \\
\hline Lying down while watching $T V$ & $\mathrm{C} 02$ & 2411 & 0.993 & 0.863 & 0.689 & 0.767 & 0.989 \\
\hline Lying down while surfing the internet & $\mathrm{C} 03$ & 79045 & 0.955 & 0.944 & 0.971 & 0.957 & 0.475 \\
\hline Lying down while sleeping & $\mathrm{C} 04$ & 2355 & 0.991 & 0.838 & 0.515 & 0.638 & 0.992 \\
\hline Sitting in a meeting & $\mathrm{C} 05$ & 4117 & 0.990 & 0.878 & 0.752 & 0.810 & 0.980 \\
\hline Sitting while watching $T V$ & $\mathrm{C} 06$ & 9246 & 0.972 & 0.820 & 0.695 & 0.752 & 0.957 \\
\hline Sitting in a car & $\mathrm{C} 07$ & 5684 & 0.979 & 0.726 & 0.715 & 0.720 & 0.973 \\
\hline Sitting while surfing the internet & $\mathrm{C} 08$ & 14443 & 0.960 & 0.782 & 0.802 & 0.792 & 0.921 \\
\hline Walking indoor & $\mathrm{C} 09$ & 2489 & 0.988 & 0.821 & 0.362 & 0.503 & 0.994 \\
\hline Walking while shopping & $\mathrm{C} 10$ & 552 & 0.996 & 0.816 & 0.185 & 0.301 & 0.999 \\
\hline Walking outdoor & $\mathrm{C} 11$ & 3727 & 0.981 & 0.622 & 0.617 & 0.620 & 0.985 \\
\hline Walking while talking & $\mathrm{C} 12$ & 2049 & 0.989 & 0.650 & 0.393 & 0.490 & 0.995 \\
\hline Standing indoor & $\mathrm{C} 13$ & 19547 & 0.915 & 0.634 & 0.800 & 0.707 & 0.888 \\
\hline Standing outdoor & $\mathrm{C} 14$ & 1399 & 0.992 & 0.740 & 0.293 & 0.420 & 0.997 \\
\hline Bicycling while exercise & $\mathrm{C} 15$ & 4953 & 0.987 & 0.845 & 0.753 & 0.797 & 0.975 \\
\hline
\end{tabular}

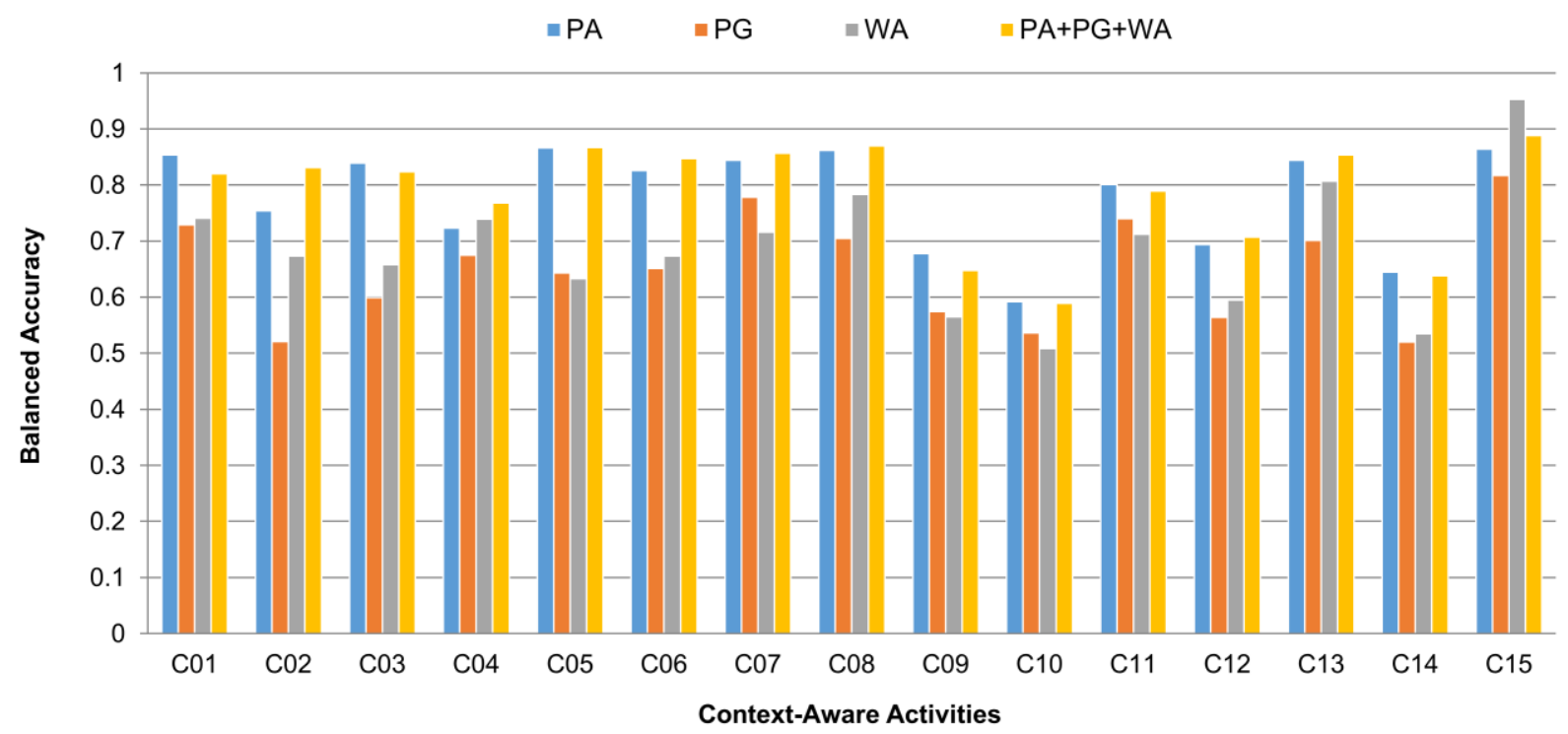

Fig. 6. Performance comparison of phone accelerometer (PA), phone gyroscope (PG), watch accelerometer (WA), and their fusion for the proposed CAHAR scheme using RF classifier. 
Fig. 6 compares the best recognition performance achieved for the proposed scheme using different sensors (phone accelerometer, phone gyroscope, watch accelerometer, and their fusion). Data pre-processing, feature selection, and accuracy evaluation procedures are adopted for the phone gyroscope and watch accelerometer in the same way as utilized for the phone accelerometer. Hence, the same parameters are used for noise filtering, data segmentation, classifier training, and validation. For feature selection, CfsSubetSel is re-evaluated on the gyroscope and watch accelerometer data individually, and the final set of features obtained is used for evaluating the selected classifiers. In the case of sensor fusion, the best-selected features for each of the individual sensors are concatenated to find out the recognition results. RF classifier provided the best BA values for each individual sensor as well as their fusion. It can be observed from Fig. 6 that the phone accelerometer provides the best individual recognition performance for most of the contextaware activities. However, for $\mathrm{C} 04$ (lying down while sleeping) and $\mathrm{C} 15$ (bicycling while exercising), the individual recognition performance of the watch accelerometer is better than the phone accelerometer. The overall average BA values attained for the proposed scheme using RF classifier are $77.9 \%, 65.1 \%, 68.6 \%$, and $78.3 \%$ using phone accelerometer, phone gyroscope, watch accelerometer, and the fusion of all three sensors, respectively. Hence, it is evident that the fusion of these sensors does not provide any significant improvement in the system accuracy when compared to BA achieved using the phone accelerometer only. Accordingly, based on the recognition accuracy of the proposed system and its computational cost, it is concluded that the proposed CAHAR method provides the best performance using a smartphone accelerometer.

In general, as compared to most of the existing HAR schemes that are practicable only under some specific settings, the proposed scheme effectively offers in-the-wild HAR. Hence, it can be used for HAR in diverse environments and varying contexts. The limitations associated with the practical use of the existing HAR systems are owing to the void of training in diverse settings and environments. Training usually involves some controlled environment, a set of rules to follow, and a pre-occupied list of activities, which affect in-the-wild user behavior. Moreover, the collection of in-the-wild data for system training is a challenging task from the practical and legal perspectives. There exist various unpretentious issues with data collection procedures due to inadequate amount and diversity of data, the sparse representation of real-world use cases, and critical self-consciousness of the participants. In addition, in-the-wild data collection and self-reporting labels from the users may result in the poor realization of the users' activities and their contexts. Nevertheless, if we can train the system based on the data related to the user's natural behavior in diverse contexts, then in-the-wild HAR can be achieved successfully. Henceforth, in this research work, we focus on training the proposed system for CAHAR based on the mobile sensor data collected in-the-wild from 60 participants in diverse contexts without entailing any scripted tasks. Hence, the proposed scheme is independent of any controlled environments and can be applied effectively to diverse contexts for HAR.

\section{CONCLUSION AND FUTURE WORK}

In this paper, we concentrate on recognizing context-aware human activities in-the-wild using the phone accelerometer. For this purpose, we select six primary daily living activities and model the user's behavioral context information with these activities to recognize fifteen context-aware activities. The experimental results indicate that the proposed scheme achieves efficient recognition performance using Random Forest classifier. Moreover, we conclude that recognizing behavioral context with the physical activity in-the-wild is a challenging task due to the inconsistencies in user behavior and activity patterns. However, incorporating a detailed user context provides a better understanding of human activity, which is very useful for human behavior analysis.

In the future, the proposed study can be extended to incorporate the user's natural activities and behavior in a large number of contexts and environments. Additional sensors and the relevant feature extraction and fusion strategies can be employed to improve system accuracy. Furthermore, emotional states of the users can be recognized based on physical activity patterns in specific contexts. In this way, a relationship can be investigated between the physical activities of a user and his/her mental state. Moreover, personalized human behavior can be modeled in different contexts, which can further be utilized for the recommender systems.

\section{ACKNOWLEDGMENT}

This research work is sponsored by the Directorate of Advanced Studies, Research and Technological Development (ASR\&TD), University of Engineering and Technology (UET), Taxila, Punjab, Pakistan.

\section{REFERENCES}

[1] G. J. Nalepa, K. Kutt, and S. Bobek, "Mobile platform for affective context-aware systems," Futur. Gener. Comput. Syst., vol. 92, pp. 490-503, 2018.

[2] S. Zhang, Z. Wei, J. Nie, L. Huang, S. Wang, and Z. $\mathrm{Li}$, "A Review on Human Activity Recognition Using Vision-Based Method," J. Healthc. Eng., vol. 2017, pp. 1-31, 2017.

[3] P. Wang, W. Li, P. Ogunbona, J. Wan, and S. Escalera, "RGB-D-based human motion recognition with deep learning: A survey," Comput. Vis. Image Underst., vol. 171, pp. 118-139, 2018.

[4] L. Köping, K. Shirahama, and M. Grzegorzek, “A general framework for sensor-based human activity recognition," Comput. Biol. Med., vol. 95, pp. 248260, 2018.

[5] M. Espinilla, J. Medina, J. Hallberg, and C. Nugent, "A new approach based on temporal sub-windows for online sensor-based activity recognition," Journal of Ambient Intelligence and Humanized Computing, pp. 1-13, 2018.

[6] M. Shoaib, S. Bosch, O. D. Incel, H. Scholten, and P. J. M. Havinga, "Complex human activity recognition using smartphone and wrist-worn motion sensors," Sensors (Switzerland), vol. 16, no. 4, 2016.

[7] Z. S. Abdallah, M. M. Gaber, B. Srinivasan, and S. 
Krishnaswamy, "Activity Recognition with Evolving Data Streams," ACM Comput. Surv., vol. 51, no. 4, pp. 1-36, 2018.

[8] H. F. Nweke, Y. W. Teh, G. Mujtaba, and M. A. Algaradi, "Data fusion and multiple classifier systems for human activity detection and health monitoring: Review and open research directions," Inf. Fusion, vol. 46, pp. 147-170, 2019.

[9] L. Cao, Y. Wang, B. Zhang, Q. Jin, and A. V. Vasilakos, "GCHAR: An efficient Group-based Context - aware human activity recognition on smartphone," J. Parallel Distrib. Comput., vol. 118, pp. 67-80, 2018.

[10] L. G. Vaizman Yonatan, Ellis Katherine, "Recognizing Detailed Human Context In-theWildfrom Smart phones and Smartwatches," IEEE Pervasive Comput., vol. 16, 2017.

[11] M. Shoaib, S. Bosch, O. Incel, H. Scholten, and P. Havinga, "A Survey of Online Activity Recognition Using Mobile Phones," Sensors, vol. 15, no. 1, pp. 2059-2085, 2015.

[12] V. Yonatan and K. Ellis, "The ExtraSensory Dataset," Data Collection In-the-Wild with Rich User Interface to Self-Report Behavior, 2016. .

[13] M. Cornacchia, K. Ozcan, Y. Zheng, and S. Velipasalar, "A Survey on Activity Detection and Classification Using Wearable Sensors," IEEE Sens. J., vol. 17, no. 2, pp. 386-403, 2017.

[14] P. Bharti, D. De, S. Chellappan, and S. K. Das, "HuMAn: Complex activity recognition with multimodal multi-positional body sensing," IEEE Trans. Mob. Comput., vol. 18, no. 4, pp. 857-870, 2019.

[15] A. R. Anwary, H. Yu, and M. Vassallo, "Gait Evaluation using Procrustes and Euclidean Distance Matrix Analysis," IEEE Journal of Biomedical and Health Informatics, 2018.

[16] F. Hussain, F. Hussain, M. Ehatisham-ul-Haq, and M. A. Azam, "Activity-Aware Fall Detection and Recognition based on Wearable Sensors," IEEE Sens. J., 2019.

[17] P. Esfahani and H. T. Malazi, "PAMS: A new position-aware multi-sensor dataset for human activity recognition using smartphones," in 2017 19th International Symposium on Computer Architecture and Digital Systems, CADS 2017, 2018, vol. 2018Janua, pp. 1-7.

[18] M. M. Hassan, M. Z. Uddin, A. Mohamed, and A. Almogren, "A robust human activity recognition system using smartphone sensors and deep learning," Futur. Gener. Comput. Syst., vol. 81, pp. 307-313, 2018.

[19] K. Lee and M. P. Kwan, "Physical activity classification in free-living conditions using smartphone accelerometer data and exploration of predicted results," Comput. Environ. Urban Syst., vol. 67, no. September 2017, pp. 124-131, 2018.

[20] N. Neverova et al., "Learning Human Identity from Motion Patterns," IEEE Access, vol. 4, pp. 18101820, 2016.

[21] M. Ehatisham-ul-haq, M. Awais, U. Naeem, Y. Amin, and J. Loo, "Continuous authentication of smartphone users based on activity pattern recognition using passive mobile sensing," J. Netw. Comput. Appl., vol. 109, no. March, pp. 24-35, 2018.

[22] J. Morales and D. Akopian, "Physical activity recognition by smartphones, a survey," Biocybern. Biomed. Eng., vol. 37, no. 3, pp. 388-400, 2017.

[23] A. Wang, G. Chen, J. Yang, S. Zhao, and C. Y. Chang, "A Comparative Study on Human Activity Recognition Using Inertial Sensors in a Smartphone," IEEE Sens. J., vol. 16, no. 11, pp. 4566-4578, 2016.

[24] A. Taherkhani, G. Cosma, A. A. Alani, and T. M. McGinnity, "Activity recognition from multi-modal sensor data using a deep convolutional neural network," in Advances in Intelligent Systems and Computing, 2019, vol. 857, pp. 203-218.

[25] E. Garcia-Ceja, C. E. Galván-Tejada, and R. Brena, "Multi-view stacking for activity recognition with sound and accelerometer data," Inf. Fusion, vol. 40, pp. 45-56, 2018.

[26] Y. Wang, S. Cang, H. Yu, and S. Member, "A Data Fusion-Based Hybrid Sensory System for Older People's Daily Activity and Daily Routine Recognition," IEEE Sens. J., vol. 18, no. 16, pp. 6874-6888, 2018.

[27] F. I. Sadiq, A. Selamat, R. Ibrahim, M. H. Selamat, and O. Krejcar, "Stampede prediction based on individual activity recognition for context-aware framework using sensor-fusion in a crowd scenarios," in Frontiers in Artificial Intelligence and Applications, 2017, vol. 297, pp. 385-396.

[28] Y. V. Mane and A. R. Surve, "CAPM: Context aware provisioning middleware for human activity recognition," in Proceedings of 2016 International Conference on Advanced Communication Control and Computing Technologies, ICACCCT 2016, 2017, pp. 661-665.

[29] A. M. Khattak et al., "Context-aware human activity recognition and decision making," in 12th IEEE International Conference on e-Health Networking, Application and Services, Healthcom 2010, 2010.

[30] F. J. Ordóñez and D. Roggen, "Deep convolutional and LSTM recurrent neural networks for multimodal wearable activity recognition," Sensors (Switzerland), vol. 16, no. 1, 2016.

[31] J. Wang, Y. Chen, S. Hao, X. Peng, and L. Hu, "Deep learning for sensor-based activity recognition: A survey," Pattern Recognit. Lett., vol. 119, pp. 3-11, 2019.

[32] H. F. Nweke, Y. W. Teh, M. A. Al-garadi, and U. R. Alo, "Deep learning algorithms for human activity recognition using mobile and wearable sensor networks: State of the art and research challenges," Expert Systems with Applications, vol. 105. pp. 233 261, 2018.

[33] C. A. Ronao and S. B. Cho, "Human activity recognition with smartphone sensors using deep learning neural networks," Expert Syst. Appl., vol. 59, pp. 235-244, 2016.

[34] N. Y. Hammerla, S. Halloran, and T. Plötz, "Deep, 
convolutional, and recurrent models for human activity recognition using wearables," in IJCAI International Joint Conference on Artificial Intelligence, 2016, vol. 2016-Janua, pp. 1533-1540.

[35] M. Ehatisham-ul-Haq et al., "Authentication of smartphone users based on activity recognition and mobile sensing," Sensors (Switzerland), vol. 17, no. 9, 2017.

[36] M. a Hall and L. A. Smith, "Feature subset selection: a correlation based filter approach," in Progress in Connectionist-Based Information Systems, Vols 1 and 2, 1997, vol. Progress i, pp. 855-858.

[37] J. C. Platt, Sequential Minimal Optimization: A Fast Algorithm for Training Support Vector Machines. 1998.

[38] S. Yao, S. Hu, Y. Zhao, A. Zhang, and T. Abdelzaher, "DeepSense: A unified deep learning framework for time-series mobile sensing data processing," in 26th International World Wide Web Conference, WWW 2017, 2017, pp. 351-360.

[39] F. Attal, S. Mohammed, M. Dedabrishvili, F. Chamroukhi, L. Oukhellou, and Y. Amirat, "Physical Human Activity Recognition Using Wearable Sensors," Sensors, vol. 15, no. 12, pp. 31314-31338, 2015.

[40] S. Scheurer, S. Tedesco, K. N. Brown, and B. O'Flynn, "Human activity recognition for emergency first responders via body-worn inertial sensors," 2017 IEEE 14th Int. Conf. Wearable Implant. Body Sens. Networks, BSN 2017, pp. 5-8, 2017.

[41] T. Zebin, M. Sperrin, N. Peek, and A. J. Casson, "Human activity recognition from inertial sensor timeseries using batch normalized deep LSTM recurrent networks," Conf. Proc. ... Annu. Int. Conf. IEEE Eng. Med. Biol. Soc. IEEE Eng. Med. Biol. Soc. Annu. Conf., vol. 2018, pp. 1-4, 2018.

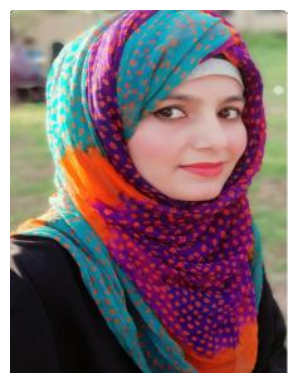

Yusra Asim received her B.Sc. degree in computer system engineering from Mirpur University of Science and Technology (MUST), AJK Pakistan, in 2017 and won Silver Medal. She is currently pursuing her M.Sc. from the University of Engineering and Technology (UET) Taxila, Pakistan. Her field of specialization is digital design, and her research domain is ubiquitous computing. Her research interests include machine learning, activity and context recognition, and human behavior analysis.

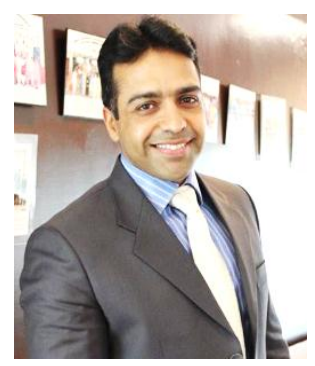

Muhammad Awais Azam received his B.Sc. degree in Computer Engineering from the University of Engineering and Technology (UET), Taxila, Pakistan and won Gold Medal in 2006, his M.Sc. degree in Wireless Networks (with distinction) from Queen Mary University, London, UK in 2008 and his $\mathrm{PhD}$ degree in Pervasive and
Ubiquitous Computing from London, UK in 2012. Between 2006 and 2007, he was a Lecturer at UET, Taxila. From May 2012 to July 2013, he worked as Head of Academics in Cromwell College of IT \& Management, London, UK. From August 2013, he has been an Assistant Professor at the Department of Computer Engineering, UET Taxila, Pakistan. He leads a research team of M.Sc. and PhD students in the area of pervasive and ubiquitous computing. His research interest includes network architecture, IoT, network security, embedded systems, ambient intelligence, wireless communications, opportunistic networks, and recommender systems.

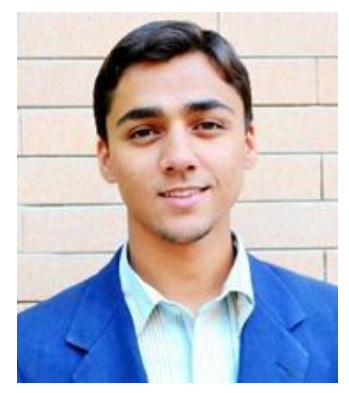

Muhammad Ehatisham-ul-Haq got his B.Sc. degree in Computer Engineering from the University of Engineering and Technology (UET), Taxila, Pakistan, in 2014 and won Gold Medal. He acquired his M.Sc. degree in Computer Engineering from UET Taxila, Pakistan, in 2017 and won Chancellor's Gold Medal. He is currently pursuing his $\mathrm{PhD}$ degree in Computer Engineering from UET Taxila, Pakistan. His field of specialization is pervasive and ubiquitous computing. His research interests are within the areas of signal, image, and video processing, biomedical signal processing, mobile sensing, machine learning, human activity and emotion recognition, and human behavior analysis.

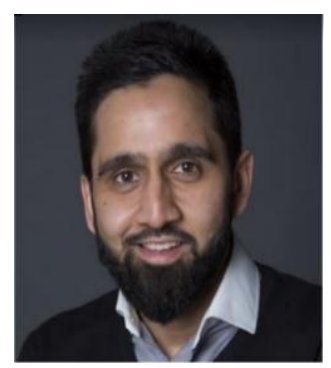

Usman Naeem is a Lecturer within the School of Electronic Engineering and Computer Science at Queen Mary University of London, UK. He was awarded his $\mathrm{PhD}$ in 2009 and 1st class B.Sc. (Hons) in Information and communication technology in 2005, both from Queen Mary University of London, UK.

His research interests are within the areas of pervasive and ubiquitous computing. Much of his research is focused on assistive technologies to support independent living for the elderly community. His other research interests also include context awareness, machine learning techniques, mobile sensing, educational technologies, gamification, and ambient intelligent environments.

Asra Khalid received her B.Sc. and M.Sc. degrees in Software Engineering from the University of Engineering and Technology (UET), Taxila, Pakistan, in 2012 and 2014 respectively. She is currently pursuing her $\mathrm{PhD}$ degree. Her areas of research interest are machine learning, recommender systems, ambient intelligence, and human activity and behavior analysis using recommender systems in smart environments. 